\title{
Near Infrared Fluorescence Enhancement by Local Surface Plasmon Resonance from Arrayed Gold Nanoblocks
}

\author{
Fuyuki Ito $^{1^{*}}$, Ryuichi Ohta ${ }^{2}$, Yukie Yokota ${ }^{3}$, Kosei Ueno $^{3}$, Hiroaki Misawa ${ }^{3}$, Toshihiko Nagamura ${ }^{2,4^{*}}$ \\ ${ }^{1}$ Department of Chemistry, Faculty of Education, Shinshu University, Nagano, Japan \\ ${ }^{2}$ Department of Applied Chemistry, Faculty of Engineering, Kyushu University, Fukuoka, Japan \\ ${ }^{3}$ Research Institute for Electronic Science, Hokkaido University, Sapporo, Japan \\ ${ }^{4}$ Department of Materials Science and Chemical Engineering, Kitakyushu National College of Technology, Fukuoka, Japan \\ Email: "fito@shinshu-u.ac.jp, ${ }^{*}$ nagamura@kct.ac.jp
}

Received November 14, 2012; revised December 14, 2012; accepted December 21, 2012

\begin{abstract}
The near infrared (NIR) fluorescence enhancement by local surface plasmon resonanoce from arrayed gold (Au) nanoblocks was investigated by NIR fluorescent dyes, IR780, immobilized in hydrophobic DNA thin film on glass substrates, to clarify the gap mode effect on the fluorescence enhancement. In the substrate with Dimer type Au nanoblock arrangement, average total fluorescence intensity was larger by 10.0, 2.4, and 12.4 times for non-polarized, P- and Spolarization as compared with that on a glass substrate alone, respectively. These findings suggested that enhancement of excitation light intensity at nanogap in the Dimer type Au nanoblock arrangement affected the fluorescence intensity. Average total fluorescence intensity, on the other hand, was smaller by 0.63 times as compared with that on a glass substrate alone in the checkerboard type Au array. It is suggested that the fluorescence quenching was caused by the energy transfer from the excited state of IR780 to Au nanoblocks or by the increased deactivation of excited dye molecules induced by resonance with Au nanoblocks at the checkerboard arrangement. We have firstly achieved the NIR fluorescence enhancement by LSPR due to the gap mode.
\end{abstract}

Keywords: Gap Mode of Gold Nanoblock Array; Fluorescence Enhancement; Near-Infrared Fluorescence Dye; Local Surface Plasmon

\section{Introduction}

Local surface plasmon from metal nano structures provides strong photon-molecule coupling fields [1], which markedly assist photochemical reaction such as photocatalysis [2], photovoltaic cell [3], photochromic reaction $[4,5]$, and optical excitation process [6]. One of the applications of the liner excitation process is the fluorescence enhancement of the dye by surface plasmon resonance [7]. The near-infrared (NIR) fluorescent dyes have several disadvantages such as low fluorescence quantum yield, low stability, and low durability compared with visible fluorescent dyes in aqueous solutions and solid films, although the NIR fluorescent dyes have been widely used in biochemical and medical fields for in vivo imaging [8]. The molecular structure of NIR dyes with extended $\pi$-conjugation is mostly responsible for these properties. The low fluorescence quantum yield is essentially governed by the Strickler-Berg relation [9]. The

*Corresponding author. energy transfer from porphyrin to NIR fluorescent dyes along the DNA double helix was investigated upon excitation at the visible region in order to overcome these disadvantages [10]. The NIR fluorescence was enhanced up to 86 times due to the efficient energy transfer from the excited porphyrin in the presence of DNA. The DNA matrix contributes not only to make efficient energy transfer, but also to improve stability and durability of NIR dye by immobilization between base pairs or in the groove. We also reported the application of confined and electric field enhanced light at surface plasmon resonance (SPR) condition to highly sensitive fluorescence detection of dyes in DNA ultrathin films deposited on a metal film and to high performance nitrogen oxides gas sensing [11]. These results demonstrated that DNA chain is one of the most powerful tools for nanoassemblies and will give novel concepts of materials design.

Fluorescence enhancement by LSPR was reported by several groups [12-15]. We have also been making efforts to enhance NIR fluorescence by LSPR from metal nanostructures using the regularly arranged Au nanoblocks 
(Au arrays) without interaction between nanostructures which show specific absorption in the NIR region [16, 17]. Average total fluorescence intensity was larger by about 1.1 to 2.5 times depending on the Array structure. The fluorescence enhancement depends on the location of LSPR absorption. We also investigated polarization dependence of excitation light for fluorescence enhancement of the NIR dye in the same systems [17]. The enhancement factor for S-polarized excitation was about 1.2 to 1.5 times as large as that for P-polarized light. The calculated enhancement factor due to the projection of horizontal direction values was identical to that due to the excitation by S-polarization, suggesting that the observed fluorescence enhancement by the Au arrays originated from the resonance of photo-electric field along the plasmon band of transverse and longitudinal mode.

In this paper, we report the enhancement of NIR fluorescence intensity using the regularly arranged $\mathrm{Au}$ nanostructures with interaction between nanostructures, which show specific absorption in the NIR region to clarify the gap mode effect on the fluorescence enhancement.

\section{Experimental}

Regularly arranged $\mathrm{Au}$ nanoblocks were fabricated by the electron beam lithography on glass plates $[18,19]$. Figure 1 shows SEM images of Au nanoblock array. The Dimer type of Au arrays (Dimer) with designed dimensions of $100 \times 100 \times 40 \mathrm{~nm}$ were prepared as shown in Figure 1(a). The gap distance of Dimer Au blocks, which is defined as the shortest distance between the nearest corners of the blocks, were $10 \mathrm{~nm}$. Checker board type of $\mathrm{Au}$ arrays (Checker) with designed dimensions of $100 \times$ $100 \times 40 \mathrm{~nm}$, whose gap is $15 \mathrm{~nm}$ as shown in Figure 1(b). As a NIR fluorescent dye, 2-[2-[2-Chloro-3-[(1,3-dihydro-3,3-dimethyl-1-propyl-2H-indol-2-ylidene)ethyli-

(a)
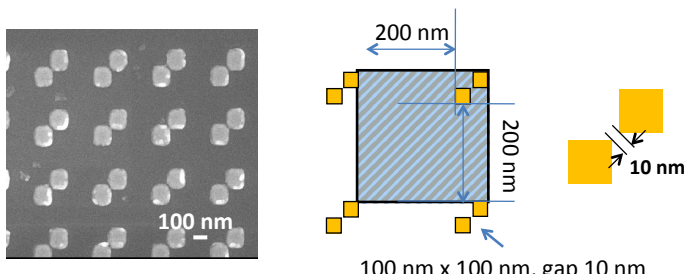

(b)
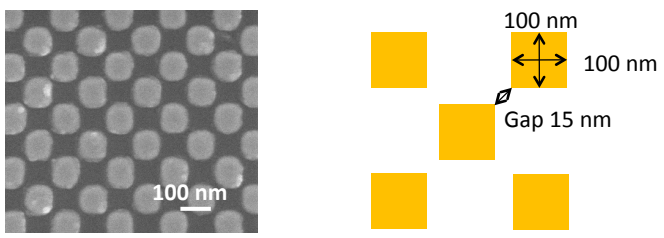

Figure 1. SEM images and schematic representation of a gold nanoblock array. (a) Dimer structure (Dimer; $100 \times$ $100 \times 40 \mathrm{~nm}$, gap: $10 \mathrm{~nm}$ ); (b) Checker board structure (Checker; $100 \times 100 \times 40$ nm, gap: $15 \mathrm{~nm}$ ). dene]-1-cyclohexen-1-yl]ethenyl]-3,3-dimethyl-1-pro-pyl indolium iodide (IR780; Aldrich Co. Ltd., Figure 2) was selected. Hydrohobic DNA (H-DNA) was prepared by exchanging sodium ions with hexadecyltrimethylammonium ions according to the reported methods [20,21]. H-DNA thin films with immobilized dyes were prepared by spin coating on glass plates with Au arrays. The film thickness was $40 \mathrm{~nm}$. UV-Vis absorption (extinction) spectra were measured by Hitachi U-4100 spectrometer. Fluorescence spectra were measured by Hitachi F-4500 fluorescence spectrophotometer. The CW laser at $675 \mathrm{~nm}$ was used as excitation light. The polarization of excitation light was controlled by a film polarizer. The incident angle for the sample was set at $40^{\circ}$.

\section{Results and Discussion}

The absorption spectrum of IR780 in H-DNA thin film shows a peak around $800 \mathrm{~nm}$ with a shoulder around 730 $\mathrm{nm}$. The fluorescence peak located at $817 \mathrm{~nm}$. The absorption (extinction) spectra of Dimer and Checker exhibit two peaks as shown in Figure 3 [18]. The extinction spectra of longitudinal (L)- and transverse (T)-modes of $\mathrm{Au}$ nanoblocks in Dimer, corresponding to the polarization of parallel and perpendicular to the block pairs, were located around 670 and $760 \mathrm{~nm}$, respectively as shown in Figure 3(a). In Checker, the extinction peak was observed around $700 \mathrm{~nm}$. The L- and T-mode is indistinguishable in Checker due to symmetric arrangement of Au nanoblocks [22].

It is difficult to make selective excitation of $\mathrm{L}$ - and T-mode in our measurement system. We attempted the Pand S-polarization dependence for the fluorescence enhancements to discuss semi-quantitatively. Figure 4(a) shows the extinction spectra of Dimer under the nonpolarized, P- and S-polarization. The extinction peaks both L- and T-mode were observed. Figures 4(b)-(d) show fluorescence spectra of IR 780 on Dimer excited at $675 \mathrm{~nm}$ with non-polarized, P- and S-polarization light, respectively. Average total fluorescence intensity was larger by 10.0, 2.4, and 12.4 times for non-polarized, P- and S-polarization as compared with that on glass substrate alone, respectively. P- and S-polarization are the vector of electric field defined as parallel and perpendicular to the plane of incidence, respectively. The schematic representation has been reported previously [17]. If the incident angle $\theta$ is not equal to zero, the P-polarization can

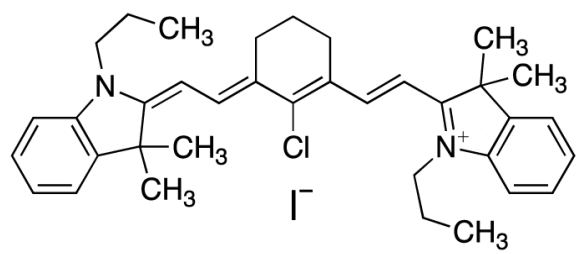

Figure 2. The molecular structure of IR $\mathbf{7 8 0}$. 

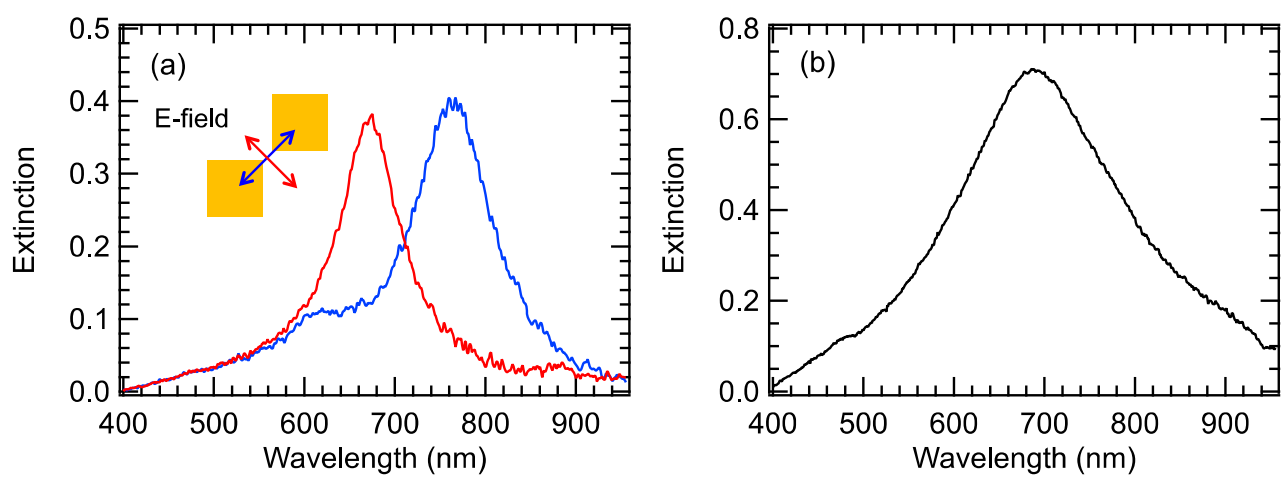

Figure 3. Extinction spectra of Dimer (a) and Checker (b); red, blue and black line indicates L-mode, T-mode and nonpolarized light excitation.
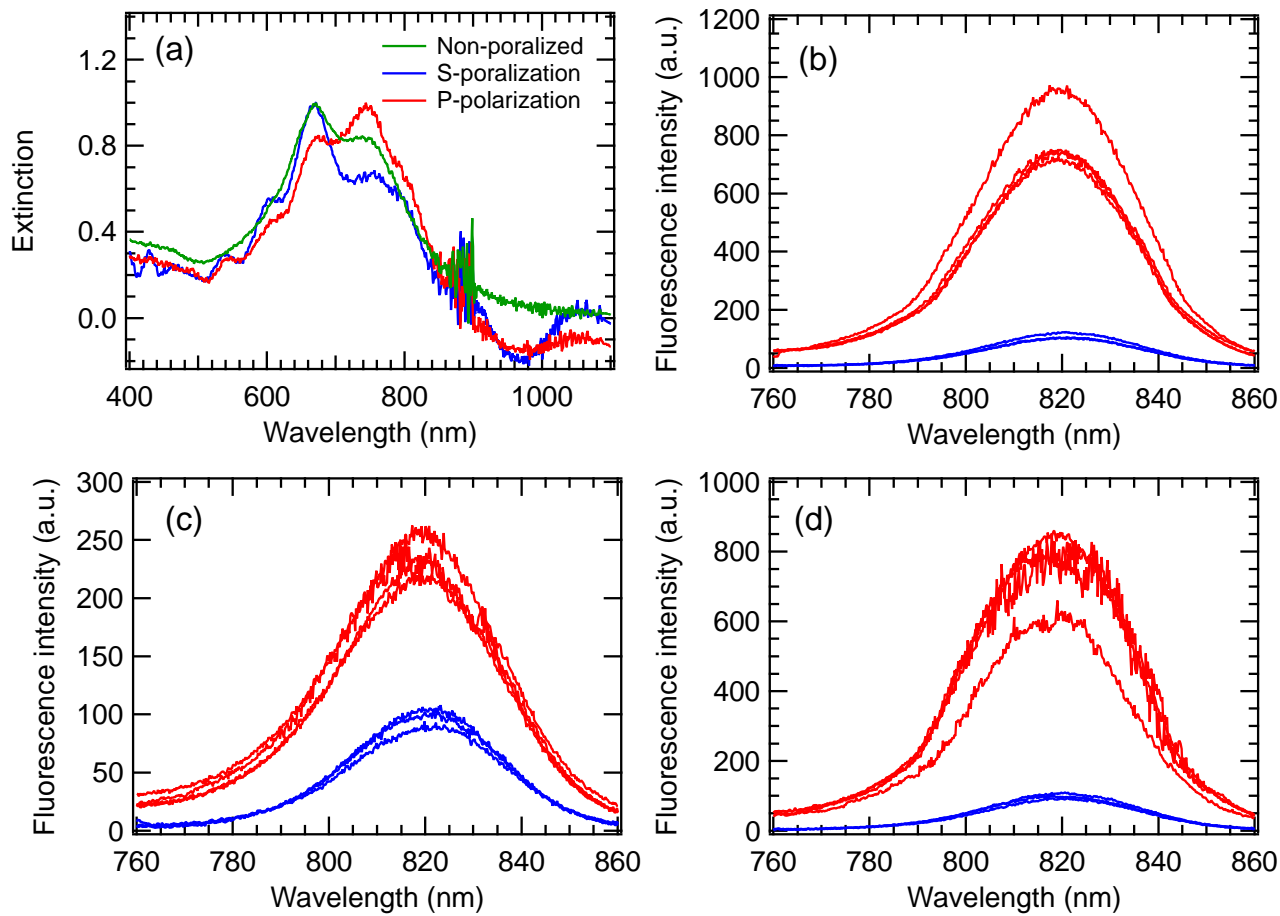

Figure 4. Extinction spectra of IR 780 in H-DNA thin film on Dimer substrate. Fluorescence spectra of IR 780 in $\mathrm{H}$-DNA on Dimer substrate excited at $675 \mathrm{~nm}$ with (b) non-, (c) P-, and (c) S-polarization in the presence (red line) and the absence (blue line) of Au blocks.

excite the plasmon band of Au nanoblocks for both vertical and horizontal direction. We have estimated the ratio of P-polarized light for horizontal and vertical direction of Au nanoblocks is estimated to be 1:0.839 from the ratio of sine to cosine in the $\theta=40^{\circ}$. S-polarization, on the other hand, can only excite for the horizontal direction. These findings indicated that the electric field intensity of S-polarized excitation light is larger than that of P-polarized one. P-polarized light contributed to the excitation of plasmon band both of horizontal and vertical direction of $\mathrm{Au}$ nanoblocks. The vertical direction corresponds to the short axis for height direction which has the absorption in visible region did not contribute to the excitation of the gap mode. The photoelectric field with parallel component along the substrate contributes to the enhancement of excitation light intensity, that is both L- and T-modes are enhanced [22]. Bek et al. were observed an enhancement of fluorescence from a fluorescent molecule, which is sandwiched between two individual gold nanoparticles, forming a hot spot of strong field enhancement [23]. These findings suggested that enhancement of excitation light intensity at nano gap in Dimer affected the fluorescence intensity.

Figure 5 shows fluorescence spectra of IR 780 on Checker excited at $675 \mathrm{~nm}$ with non-polarized light. Average total fluorescence intensity was smaller by 0.63 times as compared with that on glass substrate alone. Checker board structure caused the fluorescence quench- 


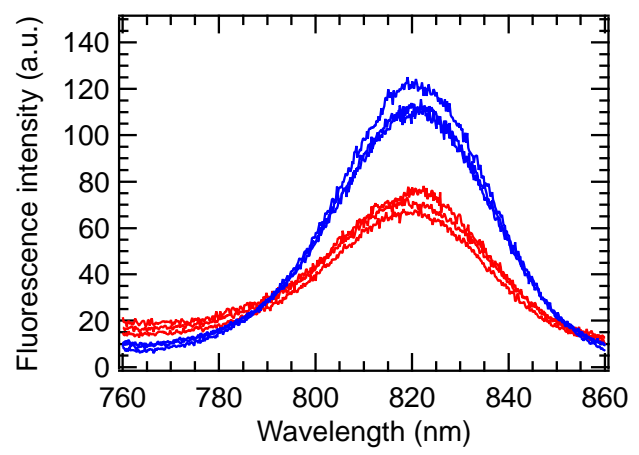

Figure 5. Fluorescence spectra of IR 780 in H-DNA on Checker substrate excited at $675 \mathrm{~nm}$ in the presence (red line) and the absence (blue line) of Au blocks.

ing rather than fluorescence enhancement, which means the fluorescence quenching originates from the energy transfer from the excited state of IR780 to Au nanoblocks or the increased deactivation of excited dye molecules induced by resonance with Au nanoblocks.

\section{Conclusion}

We investigated NIR fluorescence intensity using regularly arranged $\mathrm{Au}$ nanostructures with interaction between nanostructures in order to clarify the gap mode effect on the fluorescence enhancement. In Dimer substrate, average total fluorescence intensity was larger by for 10.0, 2.4, and 12.4 times for non-polarized, P- and Spolarization as compared with that on glass substrate alone, respectively. It is suggested that the observed fluorescence enhancement by the Au arrays most probably originated from the resonance of photo-electric field along the plasmon band in nano gap between $\mathrm{Au}$ nanoblocks of T- and L-mode. We have firstly achieved the NIR fluorescence enhancement by LSPR due to the gap mode. The fluorescence quenching, on the other hand, was observed at Checker substrate. The fluorescence quenching originates from the energy transfer from the excited state of IR780 to Au nanoblocks or the increased deactivation of excited dye molecules induced by resonance with $\mathrm{Au}$ nanoblocks rather than the fluorescence enhancement.

\section{Acknowledgements}

This work partly supported by Grant-in-Aid on Priority Area (470: Strong Photon-Molecule Coupling Fields: No. 20043027 and 21020028) from the Ministry of Education, Culture, Sports, Science, and Technology, Japan.

\section{REFERENCES}

[1] S. Y. Gao, K. Ueno and H. Misawa, "Plasmonic Antenna Effects on Photochemical Reactions," Accounts of Chemical Research, Vol. 44, No. 4, 2011, pp. 251-260. doi: $10.1021 / \operatorname{ar} 100117 \mathrm{~W}$

[2] A. Furube, L. Du, K. Hara, R. Katoh and M. Tachiya, "Ultrafast Plasmon-Induced Electron Transfer from Gold Nanodots into $\mathrm{TiO}_{2}$ Nanoparticles," Journal of the American Chemical Society, Vol. 129, No. 48, 2007, pp. 14852-14853. doi:10.1021/ja076134v

[3] Y. Tian and T. Tatsuma, "Mechanisms and Applications of Plasmon-Induced Charge Separation at $\mathrm{TiO}_{2}$ Films Loaded with Gold Nanoparticles," Journal of the American Chemical Society, Vol. 127, No. 20, 2005, pp. 7632 7637. doi:10.1021/ja042192u

[4] H. Nishi, T. Asahi and S. Kobatake, "Enhanced OnePhoton Cycloreversion Reaction of Diarylethenes near Individual Gold Nanoparticles," Journal of Physical Chemistry C, Vol. 115, No. 11, 2011, pp. 4564-4570. doi:10.1021/jp111807k

[5] Y. Tsuboi, R. Shimizu, T. Shoji and N. Kitamura, "NearInfrared Continuous-Wave Light Driving a Two-Photon Photochromic Reaction with the Assistance of Localized Surface Plasmon," Journal of the American Chemical Society, Vol. 131, No. 35, 2009, pp. 12623-12627. doi:10.1021/ja9016655

[6] S. Haruta, H. Misawa, K. Ueno, Y. Yokota, H. Uehara, H. Hiratsuka, H. Horiuchi and T. Okutsu, "Protein Crystallization Induced by Strong Photons-Molecules Coupling Fields Photochemical Reaction," Journal of Photochemistry and Photobiology A-Chemistry, Vol. 221, No. 2-3, 2011, pp. 268-272.

doi:10.1016/j.jphotochem.2011.03.012

[7] J. R. Lakowicz, "Radiative Decay Engineering: Biophysical and Biomedical Applications," Analytical Biochemistry, Vol. 298, No. 1, 2001, pp. 1-24. doi:10.1006/abio.2001.5377

[8] L. A. Cassis and R. A. Lodder, "Near-IR Imaging of Atheromas in Living Arterial Tissue," Analytical Chemistry, Vol. 65, No. 9, 1993, pp. 1247-1256. doi: $10.1021 / \mathrm{ac} 00057 \mathrm{a} 023$

[9] B. Valeur, "Molecular Fluorescence: Principles and Applications," Wiley-VCH Ltd., London, 2001.

[10] T. Kakiuchi, F. Ito and T. Nagamura, "Time-Resolved Studies of Energy Transfer from Meso-tetrakis(Nmethylpyridinium-4-yl)porphyrin to 3,3'-diethyl-2,2'-thiatricarbocyanine Iodide along Deoxyribonucleic Acid Chain," Journal of Physical Chemistry B, Vol. 112, No. 13, 1993, pp. 3931-3937. doi:10.1021/jp7107347

[11] T. Nagamura, M. Yamamoto, M. Terasawa and K. Shiratori, "High Performance Sensing of Nitrogen Oxides by Surface Plasmon Resonance Excited Fluorescence of Dye-Doped Deoxyribonucleic Acid Thin Films," Applied Physics Letters, Vol. 83, No. 4, 2003, pp. 803-805. doi:10.1063/1.1595722

[12] F. Tam, G. P. Goodrich, B. R. Johnson and N. J. Halas, "Plasmonic Enhancement of Molecular Fluorescence," Nano Letters, Vol. 7, No. 2, 2007, pp. 496-501. doi:10.1021/n1062901x

[13] N. Horimoto, K. Imura and H. Okamoto, "Dye Fluorescence Enhancement and Quenching by Gold Nanoparticles: Direct Near-Field Microscopic Observation of Shape Dependence," Chemical Physics Letters, Vol. 467, No. 
1-3, 2008, pp. 105-109. doi:10.1016/j.cplett.2008.10.067

[14] K. Sugawa, T. Kawahara, T. Akiyama, M. Kobayashi, A. Takahara and S. Yamada, "Enhanced Absorption and Emission in a Copper Phthalocyanine-Gold Nanoparticle System Assisted by Localized Surface Plasmon," Chemistry Letters, Vol. 38, No. 4, 2009, pp. 326-327. doi:10.1246/cl.2009.326

[15] G. Laurent and T. Asahi, "Enhancement of Excimer Fluorescence from Thin Dye Film by Single Gold Nanoparticles," Chemistry Letters, Vol. 38, No. 4, 2009, pp. 332333. doi:10.1246/cl.2009.332

[16] F. Ito, R. Ohta, Y. Yokota, K. Ueno, H. Misawa and T. Nagamura, "Near-Infrared Fluorescence Enhancement by Regularly Arranged Gold Nanoblocks," Chemistry Letters, Vol. 39, No. 11, 2009, pp. 1218-1219. doi:10.1246/cl.2010.1218

[17] F. Ito, R. Ohta, Y. Yokota, K. Ueno, H. Misawa and T. Nagamura, "Polarization Dependence for Enhancement of Near-Infrared Fluorescence Intensity by Local Surface Plasmon Resonance from Arranged Gold Nanoblocks," Molecular Crystals and Liquid Crystals, Vol. 538, 2011, pp. 1218-1219. doi:10.1080/15421406.2011.564085

[18] K. Ueno, V. Mizeikis, S. Juodkazis, K. Sasaki and H. Misawa, "Optical Properties of Nanoengineered Gold Blocks," Optics Letters, Vol. 30, No. 16, 2005, pp. 265-

\section{1. doi:10.1364/ol.30.002158}

[19] K. Ueno, S. Juodkazis, T. Shibuya, Y. Yokota, V. Mizeikis, K. Sasaki and H. Misawa, "Nanoparticle Plasmon-Assisted Two-Photon Polymerization Induced by Incoherent Excitation Source," Journal of the American Chemical Society, Vol. 130, No. 22, 2008, pp. 6928-6929. doi:10.1021/ja801262r

[20] K. Tanaka and Y. Okahata, "A DNA-Lipid Complex in Organic Media and Formation of an Aligned Cast Film," Journal of the American Chemical Society, Vol. 118, No. 44, 1996, pp. 10679-10683. doi:10.1021/ja9617855

[21] Y. Kawabe, L. Wang, T. Koyama, S. Horinouchi and N. Ogata, "Light Amplification in Dye-Doped DNA-Surfactant Complex Films," Proceedings of SPIE, Vol. 4106, 2000, pp. 369-376. doi:10.1117/12.408526

[22] K. Ueno, S. Juodkazis, V. Mizeikis, K. Sasaki and H. Misawa, "Clusters of Closely Spaced Gold Nanoparticles as a Source of Two-Photon Photoluminescence at Visible Wavelengths," Advanced Materials, Vol. 20, No. 1, 2008, pp. 26-30. doi:10.1002/adma.200602680

[23] A. Bek, R. Jansen, M. Ringler, S. Mayilo, T. A. Klar and J. Feldmann, "Fluorescence Enhancement in Hot Spots of AFM-Designed Gold Nanoparticle Sandwiches," Nano Letters, Vol. 8, No. 2, 2008, pp. 485-490. doi:10.1021/n1072602n 